\title{
Reflections on Palliative Care in Beijing
}

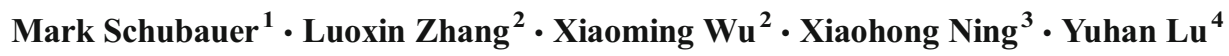

Published online: 1 August 2016

(C) American Association for Cancer Education 2016

In April 2015, I travelled to Beijing to meet with Chinese healthcare professionals who were interested in learning about hospice in the USA. The cross cultural exchange was sponsored by the International Association for Hospice and Palliative Care (IAHPC). The idea for this remarkable experience manifested a decade ago as the result of my chance meeting with Airong Lu, RN, MPH, who was in the USA for several months learning about oncology and end of life (EOL) best practices. She is now an Associate Director of Nursing at the Cancer Hospital, Chinese Academy of Medical Sciences (CHCAMS) [1].

I was guided by interpreters who were not professionally trained translators. Rather, they were young doctors and nurses who possessed a command of written and spoken English suitable to the task. Each of these individuals spoke

Mark Schubauer

akschubauer@yahoo.com

Luoxin Zhang

eugine12579@126.com

Xiaoming $\mathrm{Wu}$

xmwu03@126.com

Xiaohong Ning

ningxh1973@aliyun.com

Yuhan $\mathrm{Lu}$

lu_yuhan@sina.com

Stillharbour Healthcare Advocacy, Portland, USA

2 Cancer Hospital Chinese Academy of Medical Sciences (CHCAMS), Beijing, China

3 Peking Union Medical College, Beijing, China

4 Beijing Cancer Hospital, Beijing, China of the importance of reforming end of life care in the context of a demographic shift in the Peoples' Republic of China (PRC) that is similar to the wave of "baby boomers" in the USA [2].

I learned that palliative care is a nascent concept in Beijing and that some beginning steps are arising in oncology settings, led by pioneers who are introducing internationally accepted practices. I had the honor of meeting these change agents and what follows includes an introduction to three of them who work in elite cancer treatment centers, serving but a few of the 20 million inhabitants of an ancient, dynamic city.

The change agents have taken on an enormous challenge as have the founding members of the Beijing Living Will Promotion Association (LWPA), an organization dedicated to sowing seeds of education about how Advance Directives can improve the care of terminally ill patients [3]. Tens of thousands of people have completed advanced directives, asserting their right to refuse some life-prolonging interventions in hopes of reducing their own suffering by avoiding medically futile treatment when death is near but, "...this concept is not clearly supported by the current laws in mainland China [4]."

Nearly all deaths in Beijing occur in a hospital where reimbursement structures necessarily favor the imperative to treat over a palliative approach to care, even when death is near. (For example, daily room and board charges recover a mere fraction of the actual operating expenses at CHCAMS.) Additionally, government policies have established goals for elders to remain at home but have not yet provided for any options when it is no longer possible for elders to be safely cared for by family. I found no evidence of anything analogous to home health or durable medical equipment (DME) delivery systems that are such an integral part of US hospice care. There is an equal absence of sub-acute and/or group residential elder care programs. 
Songtang Hospice is a notable exception. The following report was prepared by Dr. Luoxin Zhang who was my principal guide and translator.

Built in 1987, Songtang was the first hospice care center in mainland China. It has admitted nearly 30,000 terminal stage patients during these years and it has relocated 7 times because neighbors thought it meant bad luck and rejected the idea of hospice. Songtang has also encountered numerous difficulties because people in China have not been educated about death and a lot of people avoid discussing death. The staff get a low wage relative to other health care professionals in Beijing because hospice care is not covered by the national health insurance. During the most recent relocation, volunteers assisted the staff in moving. Previously, the staff was unassisted, and this change may well indicate that people in China are paying more and more attention to the idea of hospice care and dying with dignity.

Songtang notwithstanding, most fragile elders are inevitably taken to an acute-care hospital where physicians and nurses become de facto hospice practitioners with little or no training in EOL care. During clinical rounds and staff education meetings, many people expressed concern and confusion about medical overtreatment and the prolongation of EOL suffering. Dozens of people asked me to comment on how to overcome the conflict that arises from implicit and explicit prohibitions that impede or prevent frank, iterative discussions about the burden and benefit of available treatments. At all 14 sites that I visited, I was asked for advice on how to speak honestly about death in a culture that places less emphasis on respect for patient autonomy and medical indications, and more emphasis on contextual features, especially the legitimate interests of families. I listened to clinicians and family members speak of the difficulty of providing emotional and psychological support to patients who, in accordance with family wishes and cultural norms, were not informed of the nature or severity of their illness.

I was struck by the familiarity of the voiced concerns and I wondered if the unexamined conceit of medical technology causes a type of suffering that transcends national boundaries and cultural norms. In both the USA and in Beijing, my response to such concerns is rooted in Elisabeth Kubler-Ross' Stages of Grief Model and this model was well received and unanimously endorsed as being culturally resonant by the Chinese citizens I spoke with.

Dr. Xiaoming Wu, Associate Director of the Comprehensive Department at CHCAMS, invited and hosted me. The Comprehensive Department serves as the de facto hospice unit at CHCAMS, admitting patients with advanced GI cancers to several large, open wards that can accommodate about 60 patients total. Dr. Wu is also the Deputy Director of the Chinese
Geriatric Oncology Society (CGOS) Palliative and Rehabilitation Committee. The timing of my trip was coordinated so that I could participate in the annual assembly of the CGOS.

Dr. Wu introduced the Good Pain Management Ward (GPMW) in 2011 as a demonstration unit at CHCAMS and it has since been recognized by the National Health and Family Planning Commission of the PRC and introduced in over 60 hospitals. The project's initial goal was the optimization of medical services for cancer patients with various pain syndromes through the standardization of the diagnosis and treatment of cancer pain by focusing on the following: educating doctors and nurses about analgesia, educating patients and their families, and ensuring the adequate supply of a comprehensive array of pain medications.

Dr. Xiaohong Ning M.D. is an Associate Professor of Gerontology at Peking Union Medical College (PUMC) and a core member of the Psychosomatic Medicine Center of Peking Union Medical College Hospital. PUMC, the most prestigious teaching hospital in the PRC, has a century long relationship with the Rockefeller Foundation's China Medical Board.

Dr. Ning visited a Taiwanese palliative care center in 2012 and was thus inspired to introduce palliative care in clinical and academic settings at PUMC. In 2014, she introduced a postgraduate class in Palliative Medicine and also started a Palliative Care consultation service. Given the size of the hospital (1800 beds), an immediate goal is to have more physicians adequately trained in order to expand the reach of the consultation service beyond what Dr. Ning can accomplish alone. As such, planning is underway to establish a program at PUMC to train physicians who are eager to learn about Palliative Care.

PUMC students with an interest in Palliative care provide a volunteer component, spending their time engaging in social interactions with patients and families on the oncology and geriatric wards. These medical student volunteers have been part of the palliative care team for almost 3 years. Research is another component of Dr. Ning's initiatives and her team is currently investigating EOL care across the Asia Pacific area. Dr. Ning is a member of numerous committees and professional associations and she is hopeful that these various groups can join together to promote Palliative Care as an urgently needed response to the care of an aging population.

Yuhan Lu, RN, MSN, the Director of Nursing at Beijing Cancer Hospital (BCH), began her career in nursing 20 years ago and was deeply affected by the unrelieved suffering of the cancer patients she cared for. When she earned a Master's Degree, she investigated the role of oncology nurses in pain management and she continues to lead research, most recently a cross-sectional survey of attitudes towards care of the dying among 280 nurses throughout China. 
$\mathrm{Lu}$ learned about the End of Life Nursing Education Curriculum (ELNEC) through her work as the Associate Director of the Oncology Nursing Committee of the Chinese Nursing Association (CNA). References to ELNEC in articles inspired her to lead a team of 12 nurses who learned and subsequently translated ELNEC into Chinese and some sections were revamped and adapted by the team to ensure cultural relevance. The main ideas of ELNEC have been incorporated into a 3-day palliative care training course sponsored annually by the Oncology Nursing Committee of the Chinese Nursing Association (CNA). In the near future, Lu hopes to develop and implement a Palliative Care specialty curriculum and certification process that will be offered through the CNA.

Chinese leaders have, over the years, successfully implemented vast reforms in health care [5] and my visit left me optimistic about the growth of Palliative Care in Beijing. In spite of an apparent lack of broad, visible support from policy makers, the people I met clearly understand the need for innovation in order to develop a twenty-first-century approach to end-of-life care that is uniquely Chinese.

\section{References}

1. http://www.jacr.info/material/iacrmeeting/a8.pdf

2. http://www.bbc.com/news/world-asia-19630110

3. http://english.caixin.com/2015-01-08/100772429.html

4. Chen HP et al (2016) Attitudes of Chinese oncology physicians towards death with dignity. J Palliat Med, Viewed online 6/24/16 http://online.liebertpub.com/doi/pdf/10.1089/jpm.2015.0344

5. Blumenthal D, Hsiao W (2015) Lessons from the east-China's rapidly evolving health care system. N Engl J Med 372:1281-1285 